\title{
Effects of Stance Width and Barbell Placement on Kinematics, Kinetics, and Myoelectric Activity in Back Squats
}

\author{
Stian Larsen ${ }^{1}$, Eirik Kristiansen ${ }^{1}$, Eric Helms ${ }^{2}$ and Roland van den Tillaar ${ }^{1,2 *}$ \\ ${ }^{1}$ Department of Sports Sciences and Physical Education, Nord University, Levanger, Norway, ${ }^{2}$ Sports Performance \\ Research Institute New Zealand, Auckland University of Technology, Auckland, New Zealand
}

OPEN ACCESS

Edited by:

Justin Keogh,

Bond University, Australia

Reviewed by:

Theodoros M. Bampouras,

Lancaster University, United Kingdom

Daniel Glassbrook,

Macquarie University, Australia

${ }^{*}$ Correspondence:

Roland van den Tillaar roland.v.tillaar@nord.no

Specialty section:

This article was submitted to Biomechanics and Control of Human Movement,

a section of the journal

Frontiers in Sports and Active Living

Received: 01 June 2021

Accepted: 02 August 2021 Published: 01 September 2021

Citation:

Larsen S, Kristiansen E, Helms E and van den Tillaar $R$ (2021) Effects of Stance Width and Barbell Placement

on Kinematics, Kinetics, and Myoelectric Activity in Back Squats. Front. Sports Act. Living 3:719013.

doi: 10.3389/fspor.2021.719013
Barbell placement and stance width both affect lifting performance in the back squat around the sticking region. However, little is known about how these squat conditions separately could affect the lifting performance. Therefore, this study investigated the effects of stance width and barbell placement upon kinematics, kinetics, and myoelectric activity around the sticking region during a three-repetition maximum back squat. Nine men and nine women (body mass: $76.2 \pm 11.1$, age: $24.9 \pm 2.6$ ) performed back squats with four different techniques, such as: high-bar narrow stance (HBNS), high-bar wide stance, low-bar narrow stance, and low-bar wide stance where they lifted $99.2 \pm 23.6$, $92.9 \pm 23.6,102.5 \pm 24.7$, and $97.1 \pm 25.6 \mathrm{~kg}$, respectively. The main findings were that squatting with a low-bar wide stance condition resulted in larger hip contributions to the total moment than the other squat conditions, whereas squatting with an HBNS resulted in greater knee contributions to the total moment together with higher vastus lateralis and less gluteus maximus myoelectric activity. Our findings suggest that training with an HBNS could be beneficial when targeting the knee extensors and plantar flexors, whereas a low-bar wide stance could be beneficial when targeting the hip extensors.

Keywords: sticking region, strength, powerlifting, electromyography, squat, inverse dynamics

\section{INTRODUCTION}

When the goal is to strengthen the lower extremities, different variations of the back squat are frequently used in resistance training (van den Tillaar and Larsen, 2020). Several studies have demonstrated that kinematics and kinetics are affected by stance width and barbell placement (Glassbrook et al., 2017, 2019). Lahti et al. (2019) investigated the effect of wide and narrow stance width upon joint kinematics, and sagittal and frontal joint moments on the hip and knees on 14 amateur rugby players at 70 and $85 \%$ of 1 repetition maximum (1-RM) in the back squat. The investigators found greater hip flexion and abduction for the wide stance width. In comparison, knee flexion was greater for the narrow stance width. When investigating the kinetics, they found that the wide stance width resulted in a greater hip extensor and knee adduction moment. In contrast, the narrow stance width resulted in a greater knee extensor moment at both 70 and $85 \%$ of 1-RM. A wide stance width has been shown in several studies to generate a different myoelectric activity profile than the narrow stance width in the back squat (Anderson et al., 1998; McCaw and Melose, 1999). Moreover, McCaw and Melose (1999) found that a wide stance width resulted in greater myoelectric activity in the adductor longus and gluteus maximus. Furthermore, as observed 
in earlier studies at maximal and near-maximal squat attempts, a sticking region occurs during the ascent (Escamilla et al., 2001; Maddox et al., 2020; Larsen et al., 2021b). This type of research often divides the ascent into three regions based on the velocity curve. The presticking region is the region between the lowest barbell height $\left(v_{0}\right)$ and the first peak in barbell velocity $\left(v_{\max 1}\right)$. The sticking region is the region between $v_{\max 1}$ and the first local minimum barbell velocity $\left(v_{\min }\right)$. Lastly, the poststicking region is the region between $v_{\min }$ and the second peak in barbell velocity $\left(v_{\max 2}\right)$ (Larsen et al., 2021b). It may therefore be of interest to athletes, practitioners, and coaches to know how squatting with different stance widths could manipulate the demands on the muscles and joint moments responsible for the sticking region because to our knowledge this has yet to be done.

The two most common back squat bar placements are the high-bar and the low-bar placement, characterized by the bar placed along the top of the upper trapezius or across the midtrapezius, over the spine of the scapulae and posterior deltoid, respectively (Glassbrook et al., 2017). Several studies have investigated the effect of barbell placements on squat performance and biomechanics (Benz and West Chester, 1989; Fry et al., 1993; Wretenberg et al., 1996; Swinton et al., 2012; Glassbrook et al., 2017, 2019). Furthermore, when squatting with loads corresponding to greater than $85 \%$ of $1-\mathrm{RM}$, a sticking region has been observed (Elliott et al., 1989). Only one study investigated the effect of barbell placement on kinematics and myoelectric activity specifically during the sticking region (van den Tillaar et al., 2020). van den Tillaar et al. (2020) investigated the effect of barbell placement upon kinematics and myoelectric activity when the barbell load was matched between the squat conditions and found no significant differences in barbell velocity, displacement, or joint angles at any of the time point "events" in and around the sticking region between the low-bar and the high-bar back squat. Moreover, they observed greater myoelectric activity for the rectus femoris, vastus medialis, and lower part of erector spinae for the high-bar position. In the sticking region, the gluteus medius and maximus myoelectric activity increased for both barbell placements, whereas myoelectric activity decreased in the quadriceps and soleus muscles. Also, the low-bar squat can result in a more anterior projection of the center of mass than the high-bar squat (Swinton et al., 2012). This may be explained by the observation that squatting with a low-bar technique often leads to greater forward lean and thereby a greater horizontal distance between the barbell and the hip joint, creating greater external hip joint moments and moment arms as speculated by van den Tillaar and Larsen (2020).

The low-bar squat is often characterized by a wider stance width and used by powerlifters, whereas the high-bar squat is often characterized by a narrow stance width and used by weightlifters (Glassbrook et al., 2019). However, to the knowledge of the authors, no works have investigated the combined effect of both stance width and barbell placement upon kinematics, kinetics, and myoelectric activity around the sticking region for different squat conditions. Therefore, this study aimed to investigate the effects of stance width and barbell placement upon the kinematics, kinetics, and myoelectric activity around the sticking region during a three-repetition maximum back squat. It was hypothesized that the low-bar conditions would produce greater hip contributions than the high-bar conditions independent of stance width, creating increased demand on the hip extensor muscles and the possibility for lifting greater loads because the low-bar squat, typically in the literature, is referred to as more hip dominant than the high-bar squat (Glassbrook et al., 2017). Also, it was hypothesized that hip moment arms and hip contribution to the total moment would peak in the sticking region independent of the squat condition.

\section{METHODS}

\section{Experimental Approach to the Research Question}

To investigate the effect of stance width and barbell placement on kinematics, kinetics, and myoelectric activity around the sticking region, a within-subjects, repeated measures design was used. Two stance widths (narrow and wide) and two barbell placements (high-bar and low-bar) were used. This resulted in four squat conditions, such as: high-bar narrow stance (HBNS), high-bar wide stance (HBWS), low-bar narrow stance (LBNS), and low-bar wide stance (LBWS) as independent variables. Dependent variables included mean myoelectric activity during the presticking, sticking, and poststicking regions, as well as net joint moments and moment arms, ground reaction forces, joint angles, barbell velocity, time, and displacement in the events $v_{0}$, $v_{\max 1}$, peak barbell deacceleration $\left(d_{\max 1}\right), v_{\min }$, and $v_{\max 2}$.

\section{Participants}

Eighteen participants who were recreationally trained lifters volunteered for this study (Table 1). Inclusion criteria were: (1) the men had to be able to lift at least 1.5 times their body mass, and women one time their own body mass in 1-RM for the preferred squat condition since men are reported to elicit greater maximal absolute strength in the lower extremity (Bishop et al., 1987); (2) none of the participants could have an injury or illness that could influence the maximal performance on the test; (3) participants had to perform the depth requirement set by the International Powerlifting Federation (IP Federation, 2019) for all squat conditions, which was that the top surface at the hip joint was below the knees in the bottom position when viewed laterally; (4) participants had to perform three familiarization sessions and two tests to be included in the analysis to ensure that they were familiar with performing all squat conditions and that the proper 3-RM was achieved in every squat condition. Written consent was obtained from all the participants before participation. The study was conducted in accordance with the latest revision of the Declaration of Helsinki and current ethical regulations for research and was approved by the National Center for Research Data (pr.nr: 701688).

\section{Procedures}

All participants participated in three familiarization sessions and two test sessions. The participants were given augmented feedback regarding the technique from two experienced powerlifting coaches during the familiarization sessions to 
ensure proper performance during test days. To prevent unnecessary exhaustion that could impact the performance, the participants had a minimum of 4 days of rest between the familiarization sessions and 7 days of rest between the test sessions.

On familiarization day one, the participants completed a questionnaire reporting their previous 1-RM and preferred stance width and signed the consent form. Thereafter height, body mass, and fat percentage were measured. Body mass and fat percentage were measured on a Tanita scale (MC-780MA, Riga, Latvia). Acromion length was measured horizontally from the right to the left acromion to decide the narrow and wide stance width for every participant, where 0.7 times the horizontal acromion length was used as a narrow stance and 1.7 times the horizontal acromion length was used as a wide stance. The stance width was marked with tape and was kept similar throughout the entire study. The participants needed to stand on the tape with the medial part of the calcaneus during the 3-RM tests in both the familiarization and test sessions. The required squat depth was marked with a horizontal band which was standardized and used for all familiarization and test sessions such that the proximal part of the hamstring had to touch the horizontal band before starting the ascent. The barbell placement for the low-bar was measured as the axial distance from the spinous process of the vertebra to the barbell (low-bar: $7.4 \pm 1.8 \mathrm{~cm}$ ). In the first familiarization session, each participant squatted up to three repetitions with $60 \%$ of predicted 3 -RM with the self-reported preferred barbell placement. During familiarization sessions two and three, participants tested a 3-RM for each of the remaining squat conditions which were not tested during the first familiarization session. 3-RM was used since it is a typical load used in training for increasing maximal strength among powerlifters. No additional guidance on how to perform the different stance widths was given. The order for the squat conditions during the familiarization and test sessions were randomized on www.randomizer.org. During familiarization 3RM testing, the repetitions in reserve-based rating of perceived exertion scale (Zourdos et al., 2016) and mean concentric barbell velocity were utilized to enhance testing accuracy. Specifically, mean concentric barbell velocity of the final repetition was recorded during each 3-RM test to ensure similar last-rep 3-RM velocities in each testing condition to ensure that true maximums were achieved. Participants had $180 \mathrm{~s}$ of rest between warm-up sets and $240 \mathrm{~s}$ of rest between test sets during all testing sessions.

On test days, electrodes for electromyography (EMG) measurements and reflective markers for the motion capture measurements were attached to the body. After a general warm-up, which included three sets of 6-10 repetitions with an unloaded Olympic barbell (Rogue, Ohio power bar), the participants performed a standardized warm-up protocol with the first squat condition. The squat protocol was as follows: four repetitions with $40 \%$ of the lowest obtained familiarization 3-RM, three repetitions with $55 \%$ of the lowest obtained familiarization 3-RM, followed by three repetitions with $70 \%$ of the lowest obtained familiarization 3-RM. The first test set started at the lowest familiarization 3-RM to ensure that the participants did not fail due to fluctuations in daily readiness and strength
(Greig et al., 2020; Larsen et al., 2021a). Thereafter, the load was increased from 1 to $10 \mathrm{~kg}$ based on the proximity to the mean concentric barbell velocity for the specific squat condition achieved in the familiarization session, or if the participant failed the third repetition. After completing each 3-RM squat condition, the participants started the next squat condition at the lowest obtained familiarization 3-RM.

\section{Recordings}

A linear encoder (ET-Enc-02, Ergotest Technology AS, Langesund, Norway) was attached to the right side of the barbell to measure vertical barbell velocity and displacement with a resolution of $0.019 \mathrm{~mm}$ and $200 \mathrm{~Hz}$ sampling rate. The barbell velocity was calculated with a five-point differential filter using Musclelab (Musclelab version: 10.200.90.5095, Ergotest innovation, Porsgrund, Norway). On the third repetition of each squat condition, the vertical barbell velocity and displacement were calculated for the following events: $v_{0}, v_{\max 1}, d_{\max 1}, v_{\min }$, and $v_{\max 2}$. Vertical ascent displacement was measured from $v_{0}$.

Musclelab (Musclelab version: 10.200.90.5095, Ergotest innovation, Porsgrund, Norway) was used to record EMG myoelectric activity of the following muscles on the dominant side of the participants: erector spinae iliocostalis, erector spinae longissimus, gluteus maximus, gluteus medius, semitendinosus, biceps femoris, adductor longus rectus femoris, vastus lateralis, vastus medialis, gastrocnemius medialis, and soleus medialis using SENIAM recommendations (Hermens et al., 2000) for location and orientation. The skin of the participants was shaved, scrubbed in alcohol, and dried with paper to reduce skin impendence before electrodes $(11 \mathrm{~mm}$ contact diameter, $20 \mathrm{~mm}$ center to center distance). They were placed on the right side of the 12 muscles with a sampling rate of $1,000 \mathrm{~Hz}$. Conductive gel (Signa Gel, Parker Laboratories INC, NJ, USA) was applied to the electrodes to reduce noise. Raw EMG signals were amplified and filtered with a preamplifier. These signals were high pass and low pass $(500,20 \mathrm{~Hz})$ filtered. The raw EMG signals were converted to the root of mean square (RMS) signals with a hardware circuit network, which had a common rejection rate of $106 \mathrm{~dB}$. The mean RMS was calculated for the presticking, sticking, and poststicking regions. For normalization, the participants performed a 5-s maximal voluntary isometric contraction (MVIC) squat at the same depth, barbell placement, and stance width as the bottom position performed with the HBNS where the barbell was mounted to a squat rack, which could be adjusted axially. The participants were instructed to obtain maximal force as quickly as possible and maintain the force throughout the trial. The mean RMS between 2.0 and $4.0 \mathrm{~s}$ was used as MVIC, whereas the mean RMS in the regions (presticking, sticking, and poststicking) was divided by the mean RMS between 2.0 and $4.0 \mathrm{~s}$ of the MVIC trial for normalization.

A three-dimensional motion capture system (Qualisys, Gothenburg, Sweden), with eight cameras at a sampling rate of $500 \mathrm{~Hz}$ and integrated force platforms, was used to track reflective markers and three-dimensional ground reaction forces. Markers were placed on both sides of the body, except for the upper and lower hand, where the markers were placed on the dominant side. Markers for the foot and shank were 
TABLE 1 | Mean \pm SD characteristics and anthropometrics of the participants.

\begin{tabular}{|c|c|c|c|c|}
\hline Physical property & Mean \pm SD & Range & Male & Female \\
\hline Age (years) & $24.9 \pm 2.6$ & $22-30$ & $26.4 \pm 2.7$ & $23.6 \pm 1.5$ \\
\hline Height (cm) & $173 \pm 8.6$ & $160-186$ & $180.1 \pm 6.8$ & $167 \pm 3.6$ \\
\hline Weight (kg) & $76.2 \pm 11.1$ & $59.6-92.4$ & $83.9 \pm 8.6$ & $69.3 \pm 8.4$ \\
\hline Fat percentage (\%) & $21.8 \pm 5.3$ & $12.9-31$ & $17.7 \pm 2.6$ & $25.4 \pm 4.4$ \\
\hline Distance from c7 low-bar (cm) & $7.4 \pm 1.8$ & $5.0-10.8$ & $8.5 \pm 1.8$ & $6.6 \pm 1.3$ \\
\hline Stance width narrow (cm) & $31.7 \pm 2.7$ & $27.6-37.0$ & $33.7 \pm 2.3$ & $29.9 \pm 1.5$ \\
\hline Stance width wide (cm) & $59.8 \pm 5.1$ & $52.2-69.9$ & $63.6 \pm 4.4$ & $56.4 \pm 2.8$ \\
\hline
\end{tabular}

TABLE 2 | Mean \pm SD load lifted for all participants, males, and females during the high-bar narrow stance, high-bar wide stance, low-bar narrow stance, and low-bar wide stance during 3-RM back squats.

\begin{tabular}{|c|c|c|c|}
\hline Squat condition & All participants (kg) & Male (kg) & Females (kg) \\
\hline High-bar narrow stance & $99.2 \pm 23.6^{\ddagger}$ & $118.6 \pm 10.9^{\ddagger}$ & $82 \pm 17.3^{\ddagger}$ \\
\hline High-bar wide stance & $92.9 \pm 23.6$ & $112.7 \pm 13.5$ & $75.3 \pm 14.6$ \\
\hline Low-bar narrow stance & $102.5 .9 \pm 24.7^{\star}$ & $122.2 \pm 10.1^{*}$ & $85 \pm 19.9^{\star}$ \\
\hline Low-bar wide stance & $95.6 \pm 25.4^{\dagger}$ & $117.7 \pm 16.7^{\dagger}$ & $78.3 \pm 15.9^{\dagger}$ \\
\hline
\end{tabular}

¥Indicates a significant difference in load lifted between the high-bar narrow stance and high-bar wide stance on a $p \leq 0.05$ level.

*Indicates a significant difference in load lifted between the low-bar narrow stance and all other squat conditions on a $p \leq 0.05$ level.

${ }^{\dagger}$ Indicates a significant difference in load lifted between the low-bar wide stance and high-bar wide stance on a $p \leq 0.05$ level.

placed on the first and fifth proximal phalanx, the lateral and medial malleolus, and the femoral lateral and medial epicondyle. Markers for the pelvis were placed on the anterior superior iliac spine and posterior superior iliac spine, creating a coda pelvis and hip joint center (Bell et al., 1987, 1990). Markers for the thorax were placed on the acromion, C7 spinous process of the vertebra, TV1 thoracal process of the vertebra, the midpoint between the inferior angles of the most caudal points of the two scapulae, sternum jugular notch, and sternum xiphisternal joint (C-Motion, 2017). Markers for the upper and lower arm segment were placed on the medial and lateral epicondyle of the humerus and the radial and ulnar styloid process. Also, four markers were placed on the barbell with a $20-\mathrm{cm}$ distance to track the events $v_{0}, v_{\max 1}, d_{\max 1}, v_{\min }$, and $v_{\max 2}$. Two force platforms (AMTI Multi-axis Force Transducer BP6001200-2000, Lexington, MA, USA; Kistler force platform, type 9260AA6, Winterthur, Switzerland) were integrated into the Qualisys motion capture system to track the three-dimensional ground reaction forces and enable inverse dynamics calculation. The origin of the axes was set to the corner of the left force platform. The $x, y$, and $z$ axes were set to mediolateral, anteriorposterior, and vertical orientations, respectively. Mediolateral and anteroposterior forces were calculated because they could result in different directions of the ground reaction force vector and cause different sagittal and frontal moments. Due to negligible values of anteroposterior forces, they were not included in the analyses.

\section{Data Analysis}

Motion capture data were exported to C3D files for segment modeling and analyses in Visual 3D v6 software (C-motion,
Germantown, MD, USA). All computations from the modelbased data were smoothed with a lowpass Butterworth filter at a cut-off frequency at $10 \mathrm{~Hz}$. Joint angles for the torso, hip, knee, and ankle in the events $v_{0}, v_{\max 1}, d_{\max 1}, v_{\min }$, and $v_{\max 2}$ were calculated in the distal to proximal orientation with a Cardan sequence in the order $x-y-z$.

Joint angles for the hip, knee, and ankle were calculated as the angle between the distal and proximal segments, and the torso angle was calculated as the angle between the torso segment and the laboratory. The three-dimensional joint moments for the hip, knee, and ankle were calculated using inverse dynamics calculations in a resolute coordinate system. The joint moments calculated in this study are internal net joint moments, expressed as means and standard deviations at events $v_{0}, v_{\max 1}, d_{\max 1 \text {, }}$ $v_{\min }$, and $v_{\max 2}$ with respect to the resolute coordinate system of the distal segments. This was calculated to observe how the joint moments changed through the ascent events. The reported net joint moments data were summed between the right and left segments. Net joint moments from the sagittal plane are flexion and extension moments, and net joint moments from the frontal plane are abduction and adduction moments. Net joint moments from the analyzed planes were normalized to the mass of the participants using default normalization and expressed as $\mathrm{Nm} / \mathrm{kg}$. When calculating the hip, knee, and ankle contributions to the total net joint moments, all abduction and adduction values were normalized into positive values.

\section{STATISTICS}

To assess differences between the sexes in the load lifted, an independent samples $t$-test was performed. For differences 
TABLE 3 | Mean \pm SD hip, knee, and ankle angles for the high-bar narrow, high-bar wide, low-bar narrow, and low-bar wide squat conditions at the events $v_{0}$, $v_{\text {max }}$, $v_{\min }$, and $v_{\max 2}$ during the back squat at 3-RM.

\begin{tabular}{|c|c|c|c|c|c|c|c|}
\hline Event & Condition & Torso angle $\left(^{\circ}\right)$ & Hip flexion $\left({ }^{\circ}\right)$ & Hip abduction $\left({ }^{\circ}\right)$ & Hip external rotation $\left({ }^{\circ}\right)$ & Knee flexion $\left(^{\circ}\right)$ & Ankle plantar flexion $\left({ }^{\circ}\right)$ \\
\hline \multirow[t]{4}{*}{$v_{0}$} & High-bar narrow & $46.7 \pm 2.9$ & $111 \pm 7.2$ & $-13.5 \pm 8.6^{\star}$ & $7.2 \pm 5.5^{*}$ & $126.4 \pm 4.7^{\ddagger}$ & $106.0 \pm 4.5^{\dagger}$ \\
\hline & High-bar wide & $46.0 \pm 3.4$ & $110.4 \pm 4.5$ & $-24.1 \pm 7.0^{\dagger^{\star}}$ & $15.7 \pm 5.2^{{\dagger^{\star}}^{*}}$ & $119.3 \pm 6.4$ & $98.6 \pm 5.9$ \\
\hline & Low-bar narrow & $56.7 \pm 2.5^{\ddagger}$ & $110.5 \pm 6.6$ & $-11.5 \pm 7.2$ & $4.0 \pm 5.9$ & $122.7 \pm 5.0^{\dagger}$ & $106.3 \pm 5.0^{\dagger}$ \\
\hline & Low-bar wide & $53.0 \pm 4.4^{\star}$ & $111.9 \pm 4.8$ & $-20.8 \pm 8.9^{\dagger}$ & $14.2 \pm 6.4^{\dagger}$ & $120.3 \pm 5.8$ & $100.5 \pm 6.3$ \\
\hline \multirow[t]{4}{*}{$V_{\max 1}$} & High-bar narrow & $51.0 \pm 3.6 \downarrow$ & $106.8 \pm 6.0 \downarrow$ & $-9.4 \pm 8.6 \downarrow^{*}$ & $7.1 \pm 5.8^{*}$ & $114.7 \pm 5.4 \downarrow \ddagger$ & $102.0 \pm 4.1^{\downarrow \dagger}$ \\
\hline & High-bar wide & $48.4 \pm 2.8 \downarrow$ & $108.7 \pm 4.8 \downarrow$ & $-20.6 \pm 6.5^{\downarrow \dagger^{\star}}$ & $13.6 \pm 5.2^{\dagger^{*}}$ & $111.1 \pm 5.6 \downarrow$ & $96.8 \pm 5.4 \downarrow$ \\
\hline & Low-bar narrow & $61.0 \pm 2.6^{\downarrow \ddagger}$ & $107.1 \pm 6.6 \downarrow$ & $-7.9 \pm 6.5 \downarrow$ & $4.6 \pm 6.1$ & $111.9 \pm 6.2 \downarrow$ & $103 \pm 5.0^{\downarrow \dagger}$ \\
\hline & Low-bar wide & $57.4 \pm 5.2 \downarrow^{*}$ & $108.3 \pm 6.1 \downarrow$ & $-16.8 \pm 8.5^{\downarrow \dagger}$ & $11.8 \pm 5.7^{\dagger}$ & $108.8 \pm 5.3 \downarrow$ & $97.4 \pm 6.0 \downarrow$ \\
\hline \multirow[t]{4}{*}{$d_{\max 1}$} & High-bar narrow & $53.2 \pm 2.8 \downarrow$ & $100.5 \pm 9.9 \downarrow$ & $-7.0 \pm 8.7^{\star}$ & $5.6 \pm 5.9 \downarrow$ & $99.9 \pm 9.2^{\downarrow \ddagger}$ & $97.3 \pm 6.5^{\downarrow \dagger}$ \\
\hline & High-bar wide & $52.3 \pm 3.1 \downarrow$ & $102.5 \pm 7.2 \downarrow$ & $-16.5 \pm 6.5^{\dagger^{\star}}$ & $9.8 \pm 5.9^{\downarrow \dagger}$ & $96.1 \pm 8.7 \downarrow$ & $91.6 \pm 6.3 \downarrow$ \\
\hline & Low-bar narrow & $63.5 \pm 3.2^{\downarrow \neq}$ & $100.2 \pm 9.6 \downarrow$ & $-5.0 \pm 6.6$ & $3.7 \pm 5.8 \downarrow$ & $95.6 \pm 9.9 \downarrow$ & $97.4 \pm 6.4^{\downarrow \dagger}$ \\
\hline & Low-bar wide & $60.7 \pm 4.8 \downarrow^{*}$ & $102.1 \pm 9.1 \downarrow$ & $-14.3 \pm 8.1^{\dagger}$ & $9.5 \pm 6.0^{\downarrow \dagger}$ & $95.5 \pm 6.5 \downarrow$ & $93.1 \pm 6.9 \downarrow$ \\
\hline \multirow[t]{4}{*}{$v_{\min }$} & High-bar narrow & $54.5 \pm 3.2$ & $92.5 \pm 9.1 \downarrow$ & $-5.9 \pm 8.1^{\star}$ & $3.3 \pm 4.7 \downarrow$ & $88.1 \pm 8.1^{\downarrow \neq}$ & $94.1 \pm 6.2^{\downarrow \dagger}$ \\
\hline & High-bar wide & $52.3 \pm 4.9$ & $96.1 \pm 8.7 \downarrow$ & $-15.0 \pm 6.3^{\dagger^{\star}}$ & $6.6 \pm 5.7^{\downarrow \dagger}$ & $86.9 \pm 8.7 \downarrow$ & $89.6 \pm 6.2 \downarrow$ \\
\hline & Low-bar narrow & $63.3 \pm 5.0^{\star}$ & $94.1 \pm 10.2 \downarrow$ & $-4.4 \pm 6.3$ & $2.3 \pm 6.2 \downarrow$ & $84.9 \pm 5.6 \downarrow$ & $93.8 \pm 6.3^{\downarrow \dagger}$ \\
\hline & Low-bar wide & $62.6 \pm 7.4^{\star}$ & $96.2 \pm 10.1 \downarrow$ & $-12.7 \pm 7.0^{\dagger}$ & $5.6 \pm 5.7^{\downarrow \dagger}$ & $82.6 \pm 4.9 \downarrow$ & $89.4 \pm 6.7 \downarrow$ \\
\hline \multirow[t]{4}{*}{$v_{\max 2}$} & High-bar narrow & $31.3 \pm 3.2 \downarrow$ & $46.5 \pm 10.0 \downarrow$ & $-7.0 \pm 5.4$ & $-9.8 \pm 6.0 \downarrow$ & $50.2 \pm 5.7 \downarrow^{*}$ & $87.4 \pm 5.0^{\downarrow \dagger}$ \\
\hline & High-bar wide & $28.6 \pm 4.4 \downarrow$ & $48.7 \pm 7.2 \downarrow$ & $-14.6 \pm 3.6^{\dagger}$ & $-11.1 \pm 6.5 \downarrow$ & $50.1 \pm 6.6 \downarrow^{\star}$ & $83.7 \pm 5.4 \downarrow$ \\
\hline & Low-bar narrow & $41.5 \pm 4.9 \downarrow^{*}$ & $52.1 \pm 9.5 \downarrow^{*}$ & $-5.7 \pm 3.1^{\dagger}$ & $-9.3 \pm 6.8 \downarrow$ & $48.3 \pm 6.7 \downarrow$ & $85.3 \pm 5.0 \downarrow$ \\
\hline & Low-bar wide & $39.3 \pm 4.5 \downarrow^{*}$ & $53.5 \pm 7.9 \downarrow^{*}$ & $-12.7 \pm 5.3^{\dagger}$ & $-9.4 \pm 7.0 \downarrow$ & $47.0 \pm 6.5 \downarrow$ & $81.8 \pm 5.2 \downarrow$ \\
\hline
\end{tabular}

Torso angles are relative to a lab as a reference segment.

$\downarrow$ Indicates a significant difference in torso angle between this event and all other events on a $p \leq 0.005$ level.

*Indicates a significant difference between this squat condition and all other barbell placements on a $p \leq 0.05$ level.

${ }^{\dagger}$ Indicates a significant difference between this squat condition and all other stance widths on a $p \leq 0.05$ level.

${ }_{\ddagger}$ Indicates a significant difference between this squat condition and all other squat conditions a $p \leq 0.05$ level.

between sexes in barbell kinematics, a repeated 2 (sex: male, female) $\times 2$ (stance width: narrow, wide) $\times 2$ (barbell placement: high-bar, low-bar $) \times 5$ (event: $v_{0}, d_{\max 1} v_{\max 1}, v_{\min }$, and $\left.v_{\max 2}\right)$ analysis of variance was performed (ANOVA). To assess the difference in the load lifted and the joint angular velocities, together with their timings between the two stance widths and barbell placement, a repeated 2 (stance width: narrow, wide) $\times 2$ (barbell placement: high-bar, low-bar) ANOVA (two-way ANOVA) was performed. For torso angles, ground reaction forces, net joint moments, moment contributions to total moment, and moment arms, a repeated 2 (stance width: narrow, wide) $\times 2$ (barbell placement: high-bar, low-bar $) \times 5$ (event: $v_{0}, d_{\max 1} v_{\max 1}, v_{\min }$, and $v_{\max 2}$ ) ANOVA was performed. For myoelectric activity, a repeated 2 (stance width: narrow, wide) $\times 2$ (barbell placement: high-bar, low-bar) $\times 3$ (regions: presticking, sticking, and poststicking) ANOVA was performed. Bonferroni post-hoc tests were used to identify where potential differences in barbell kinematics, joint kinematics, myoelectric activity, and joint kinetics occurred. If the assumption of sphericity was violated, the Greenhouse-Geisser adjustments of $p$-values were reported. All results are presented as mean $\pm \mathrm{SDs}$. Effect sizes were evaluated with $\eta_{\mathrm{p}}^{2}$ (partial eta squared), where $<0.01-0.06$ constitutes a small effect, $<0.06-0.14$ a medium effect, and $>0.14$ a large effect (Cohen, 1988). The alpha level of significance was set at $p<0.05$. Statistical analyses were conducted in SPSS version 27.0 (IBM Corp. Armonk, NY, USA).

\section{Results}

A significant effect ( $F \geq 28.86, p \leq 0.001, \eta^{2} \geq 0.51$ ) of barbell placement and stance width was found for the load lifted, where squatting with a narrow stance width allowed for more load to be lifted ( $p \leq 0.001)$. Moreover, squatting with a low-bar placement allowed the participants to squat with a greater load independent of stance width $(p \leq 0.001)$. Men lifted significantly more load ( $p$ $\leq 0.001$ ) than women during all squat conditions, but followed the same pattern of loads lifted for the different squat conditions (see Table 2).

No significant effect $\left(F \leq 2.84, p \geq 0.108, \eta^{2} \leq 0.12\right)$ was found between sexes in barbell kinematics. Descent displacement was $0.64 \pm 0.05,0.6 \pm 0.04,0.63 \pm 0.05$, and $0.61 \pm 0.05 \mathrm{~m}$ for the HBNS, HBWS, LBNS, and LBWS, respectively, whereas squatting with a narrow stance resulted in a greater descent displacement than the wide stance widths ( $p<0.001$; see Table 3 ).

For the occurrence and timing of the events, a significant effect was found for barbell placement at $v_{\max 2}(F=5.8, p=0.035$, $\eta^{2}=0.34$; see Figure 1). Post-hoc tests revealed that $v_{\max 2}$ occurred earlier for the low-bar conditions $(p=0.035)$. Also, a significant interaction effect for barbell placement with stance width and event was found at every event $(F \geq 4.8, p \leq 0.05$, $\eta^{2} \geq 0.30$ ) for barbell displacement from $v_{0}$, where the HBWS condition occurred at a lesser displacement compared with all the other squat conditions. A significant effect of stance width upon velocity $\left(F=11.67, p=0.003, \eta^{2}=0.37\right.$ ) was found 

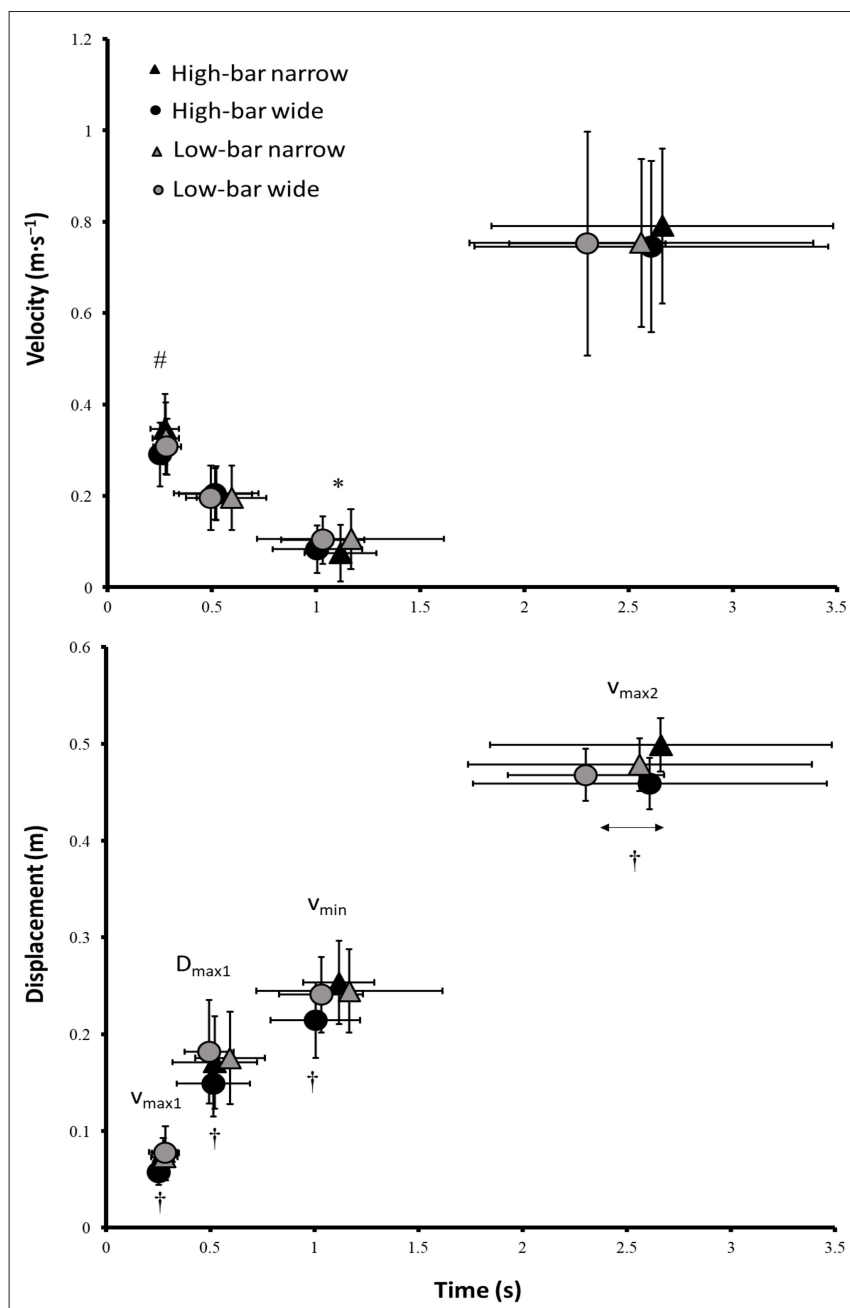

FIGURE 1 | Mean \pm SD velocity and displacement of the events $v_{\max 1}, d_{\max 1}$ $v_{\min }$, and $v_{\max 2}$, and their timing. \# Indicates a significant difference in velocity between the narrow and wide stance widths on a $p \leq 0.05$ level. * Indicates a significant difference in velocity between HBNS and LBNS on a $p \leq 0.05$ level. † Indicates a significant difference in displacement between the HBWS and all other squat conditions on a $p \leq 0.05$ level. $\leftrightarrow$ Indicates a significant difference in timing between the high-bar and low-bar barbell placements on a $p \leq 0.05$ level. in $v_{\max 1}$ together with a significant effect $(F=5.43, p=0.03$, $\left.\eta^{2}=0.21\right)$ of barbell placement in $v_{\text {min }}$. Post-hoc tests showed that barbell velocity was greater in $v_{\max }$ for the narrow stance widths compared to the wide stance widths $(p=0.003)$. At the same time, in $v_{\text {min }}$, barbell velocity was greater when squatting with a low-bar condition compared with the high-bar conditions ( $p=0.03$; see Figure 1).

A significant effect of event, stance width, and barbell placement was found for torso angle $\left(F \geq 8.09, p \leq 0.036, \eta^{2}\right.$ $\geq 0.62$; see Table 3). Post-hoc tests revealed that forward lean increased from $v_{0}$ and $v_{\max 1}$ to $d_{\max 1}$ and $v_{\min }$, respectively, before decreasing in $v_{\max 2}$ for all squat conditions. Furthermore, squatting with a low-bar technique resulted in a greater horizontal torso angle than squatting with a high-bar technique $(p=0.003)$ in all events.

A significant interaction effect between event, barbell placement, and stance width was found for hip flexion and hip abduction angles $\left(F \geq 5.8, p \leq 0.005, \eta^{2} \geq 0.35\right)$. Here $v_{\max 2}$ occurred at a greater hip flexion angle for the low-bar conditions compared with the high-bar conditions ( $p=0.003$; see Table 3 ). Post-hoc tests also showed that greater hip abduction angles were created at $v_{0}$, which decreased to $v_{\max 1}$ before remaining stable in the three last events $(p \geq 0.116)$. Furthermore, greater hip abduction angles were observed in all events for the wide stance width for the high-bar placement $(p \leq 0.034)$. Also, a significant interaction effect between event, stance width, and barbell placement occurred for hip internal rotation angles $(F \geq$ 5.4, $p \leq 0.012, \eta^{2} \geq 0.33$ ). Hip internal angles decreased from $v_{0}$ to $d_{\max 1}$ and $v_{\min }$ before changing to hip external rotation angles in $v_{\max 2}$. Furthermore, squatting with a wide stance width created a greater hip internal rotation angle at $v_{0}, v_{\max 1}, d_{\max 1}$, and $v_{\min }$ than the narrow stance widths.

Also, a significant interaction effect for event, barbell placement, and stance width was found for knee flexion and plantar flexion angles $\left(F \geq 2.72, p \leq 0.041, \eta^{2} \geq 0.20\right)$, where squatting with a narrow stance width resulted in greater knee flexion and plantar flexion angles.

The knee and ankle reached peak angular velocity at two distinct points, and their velocity decreased between these points. However, peak hip angular velocity occurred only once (see Figure 2). No significant effects of squat condition were found for hip extension velocity $\left(F=0.51, p=0.489, \eta^{2}=0.05\right)$, whereas a significant interaction between barbell placement and stance width occurred at the first maximum knee angular velocity and a significant effect of barbell placement at the second maximum knee angular velocity $\left(F \geq 6.2, p \leq 0.03, \eta^{2} \geq 0.36\right)$. At the first maximum angular velocity, the LBWS showed a greater peak knee extension angular velocity than the other squat conditions. At the second maximum angular velocity, the high-bar conditions produced a greater peak knee extension angular velocity than the low-bar conditions $(p=0.03)$. For the ankle, a significant effect occurred only at the second maximum angular velocity $\left(F=7.5, p=0.019, \eta^{2}=0.4\right)$, where the highbar conditions created a greater peak plantar flexion angular velocity than the low-bar conditions $(p=0.019)$. Also, at the second maximum angular velocity, a significant effect of barbell placement was found for every joint $\left(F \geq 5.2, p \leq 0.043, \eta^{2} \geq\right.$ 0.32 ), where the high-bar conditions produced peak hip, knee, and ankle angular velocities later in the ascent than the low-bar conditions ( $p \leq 0.043$; see Figure 2).

A significant interaction effect was found between event and barbell placement for vertical ground reaction force $(F \geq$ 12.8, $p \leq 0.006, \eta^{2} \geq 0.59$; see Figure 3), whereas the vertical ground reaction forces decreased from $v_{0}$ to all other events for all squat conditions $(p \leq 0.001)$. Also, ground reaction forces decreased from $v_{\max 1}$ to $d_{\max 1}$ before increasing in $v_{\min }$ again for all squat conditions ( $p \leq 0.014$ ), producing similar ground reaction forces at the events $v_{\max 1}, v_{\min }$, and $v_{\max 2}$. The lowbar conditions produced greater ground reaction forces than the high-bar conditions at $v_{0}$ and $v_{\max 2}$, whereas the LBNS resulted 


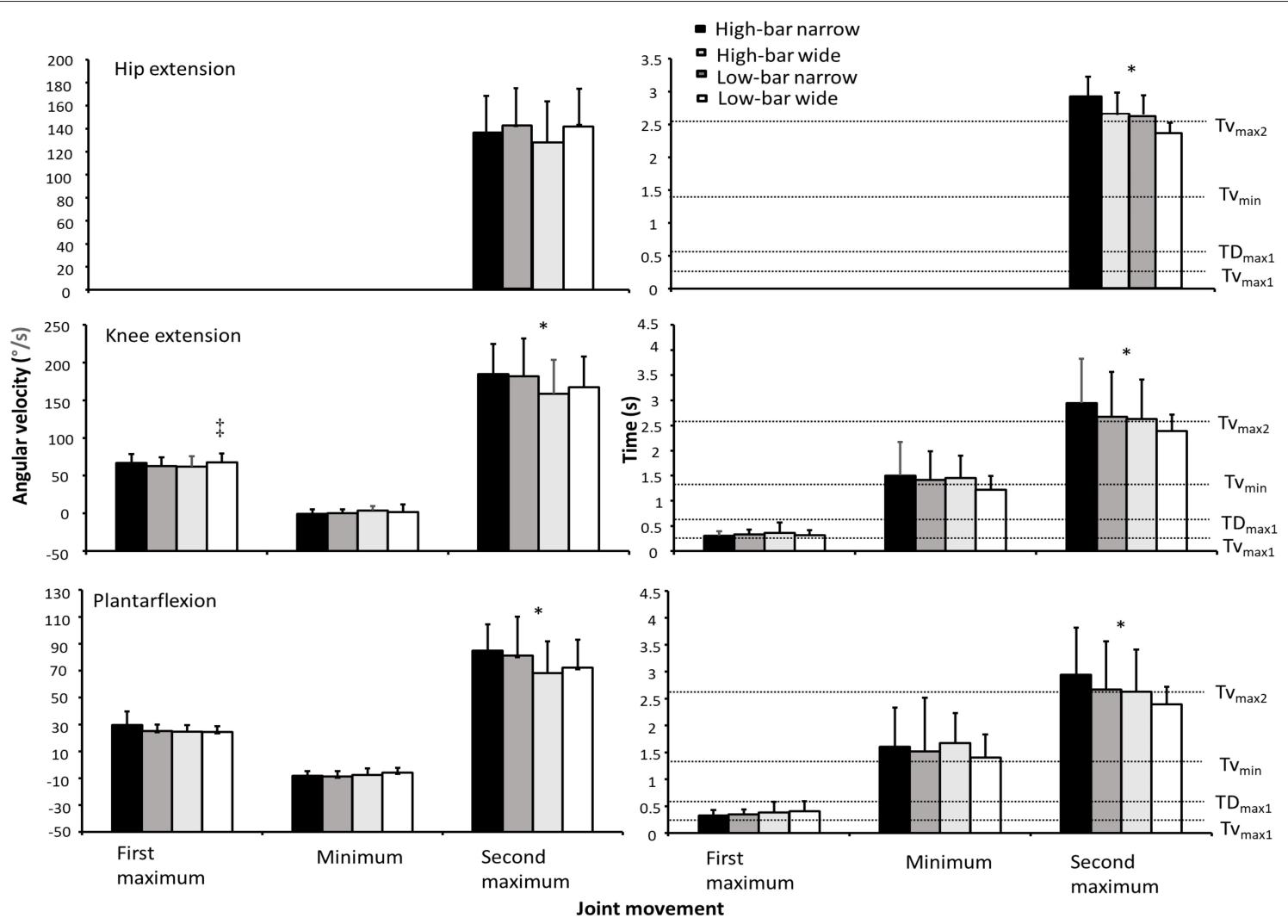

FIGURE 2 | Mean \pm SD joint movements at the hip, knee, and ankle joint together with their timings for the HBNS, HBWS, LBNS, and LBWS during 3-RM back squats. Also mean timing of the events $v_{0}, v_{\max 1}, d_{\max 1}, v_{\min }$, and $v_{\max 2}$ for all squat conditions relative to the timing of the joint movements. ${ }^{*}$ Indicates a significant difference between high-bar and low-bar barbell placements for this event on a $p \leq 0.05$ level. $\neq$ Indicates a significant difference between this squat condition and all other squat conditions for this event on a $p \leq 0.05$ level.

in greater vertical ground reaction forces than all other squat conditions at $v_{\max 1}, d_{\max 1}$, and $v_{\min }$ (see Figure 3 ).

For mediolateral ground reaction forces, a significant effect of event and stance width was found ( $\mathrm{F} \geq 25.6, p \leq 0.001$, $\eta^{2} \geq 0.72$ ), where the medially-directed ground reaction forces increased from $v_{0}$ to all other events and from $v_{\max 1}$ to $v_{\min }(p \leq 0.003)$. Hence, the wide stance widths created greater medial ground reaction forces than the narrow stance widths $(p=0.001)$. There was a significant interaction effect of event and stance width for the mediolateral/vertical force ratio $\left(F \geq 3.6, p \leq 0.045, \eta^{2} \geq 0.27\right)$ where the mediolateral to vertical force ratio was greater during all events for the wide stance widths compared with the narrow stance widths $(p=0.001)$. Furthermore, the mediolateral to vertical force ratio increased from $v_{0}$ to all events and from $v_{\max 1}$ to $v_{\min }$ and $v_{\max 2}(p \leq 0.014)$ for all squat conditions.

Significant interaction effects for event, barbell placement, and stance width were observed ( $\left.F \geq 3.8, p \leq 0.034, \eta^{2} \geq 0.26\right)$ for hip extension and plantar flexion moments. Further, a significant interaction effect for event, barbell placement, and stance width was found for knee extension moments $\left(F \geq 3.5, p \leq 0.038, \eta^{2} \geq\right.$ 0.18; see Figure 4).
The Bonferroni post-hoc tests revealed that the hip and knee extension, together with plantarflexion moments, decreased from $v_{0}$ to all other events $(p \leq 0.048)$. Hip extension moments were stable in $v_{\max 1}, d_{\max 1}$, and $v_{\min }$ before decreasing in $v_{\max 2}$ $(p=0.001)$. Additionally, squatting with a LBNS created a greater hip extension moment in all events compared to the high-bar conditions, where the LBWS demonstrated a greater hip extension moment than the high-bar conditions in $d_{\max 1}$, $v_{\min }$, and $v_{\max 2}$. Squatting with a narrow stance created the greatest knee extension moments in $v_{0}, v_{\max 1}$, and $d_{\max 1}$, but the knee extension moments during $v_{\min }$ and $v_{\max 2}$ were significantly lower during LBNS squats compared with the other squat conditions.

For the hip, knee, and ankle frontal plane moments, a significant interaction effect between event and stance width was found ( $F \geq 5.4, p \leq 0.007, \eta^{2} \geq 0.33$; see Figure 4), where hip adduction moments were created in $v_{0}$ and $v_{\max 1}$, which changed to hip abduction moments in $d_{\max 1}, v_{\min }$, and $v_{\max 2}$ for all squat conditions. Furthermore, knee abduction moments were created in $v_{0}$ and $v_{\max 1}$, which changed to knee adduction moments in $d_{\max 1}, v_{\min }$, and $v_{\max 2}$ for all squat conditions. Greater knee abduction moments were created in $v_{0}$ and $v_{\max 1}$ for the narrow squat conditions, whereas greater knee adduction moments were 


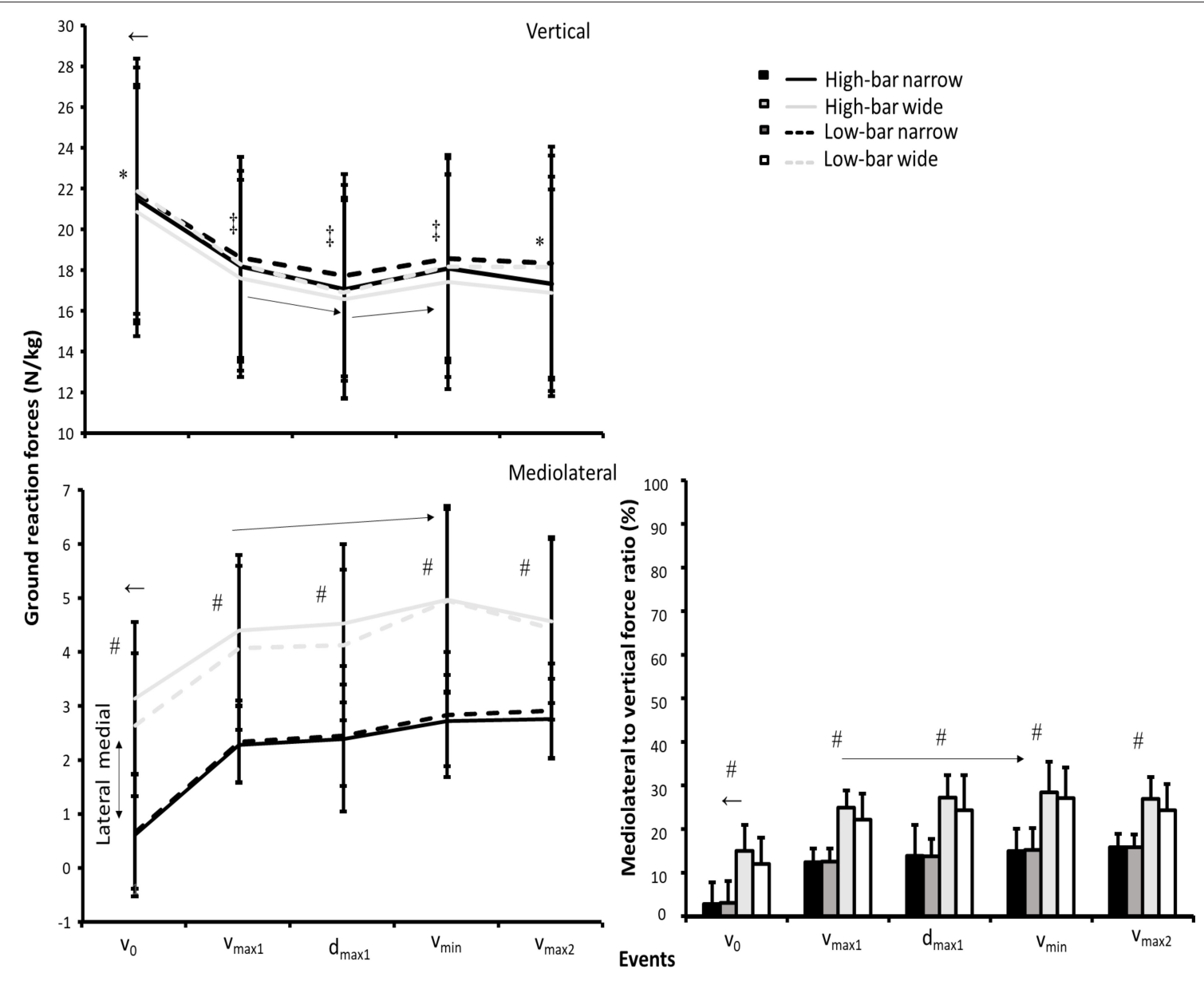

FIGURE 3 | Mean \pm SD vertical and mediolateral ground reaction force together with mediolateral to vertical force ratio normalized by body mass for the HBNS, HBWS, LBNS, and LBWS in the events $v_{0}, v_{\max 1}, d_{\max 1}, v_{\min }$, and $v_{\max 2}$ during 3-RM back squats. $\longleftarrow$ Indicates a significant difference between this event and all other events on a $p \leq 0.05$ level for each squat condition. * Indicates a significant difference between high-bar and low-bar barbell placements for this event on a $p \leq$ 0.05 level. $\neq$ Indicates a significant difference between the LBNS squat condition and all other conditions for this event on a $p \leq 0.05$ level. $\rightarrow$ Indicates a significant difference between the two events from the start to the end of the arrow on a $p \leq 0.05$ level for each squat condition. \# Indicates a significant difference between the narrow and wide stance widths for this event on a $p \leq 0.05$ level.

created for the wide stance widths in $d_{\max 1}, v_{\min }$, and $v_{\max 2}$. Also, squatting with a narrow stance width created ankle abduction moments, whereas squatting with a wide stance width created ankle adduction moments during all events.

Significant interaction effects for event and barbell placement were found for hip and knee moment arm ground reaction forces ( $F \geq 2.8, p \leq 0.035, \eta^{2} \geq 0.19$ ). Additionally, a significant interaction effect for stance width and event was found for the ankle moment arm $\left(F=3.2, p=0.022, \eta^{2} \geq 0.22\right)$. Post-hoc tests revealed that the hip moment arm increased from $v_{0}$ to $d_{\max 1}$ and $v_{\min }$ before decreasing in $v_{\max 2}(p \leq 0.025)$ for all squat conditions. Similar moment arms were produced at the first four events between conditions, however, the low-bar conditions demonstrated greater moment arms at $v_{\max 2}$ than the high-bar conditions (see Figure 5).

Knee moment arms decreased significantly at each event,

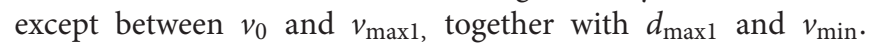

However, squatting with a narrow stance width showed greater knee moment arms in $v_{0}$ before decreasing in the subsequent events. Also, the HBWS knee moment arm increased from $v_{0}$ to $v_{\max 1}$, whereas knee moment arms decreased between these events for the other squat conditions reflected by the barbell placement, stance width, and event interaction. Greater knee moment arms were demonstrated at $v_{\max 2}$ during high-bar conditions than low-bar conditions.

For the total moment contribution, a significant effect of event, barbell placement, and stance width was found for the hip knee and ankle joints ( $F \geq 2.8, p \leq 0.043, \eta^{2} \geq 0.24$; see Figure 6).

Post-hoc tests showed that hip contribution increased from $v_{0}$ to $d_{\max 1}$ and $v_{\min }$ before decreasing at $v_{\max 2}$ for all squat conditions $(p \leq 0.023)$. Furthermore, the LBWS had the greatest hip contribution to the total moment at $v_{0}, v_{\max 1}$, and $d_{\max 1}$, before $v_{\min }$ and $v_{\max 2}$, where the LBNS showed a similar hip contribution. Also, squatting with an HBNS produced lower hip 


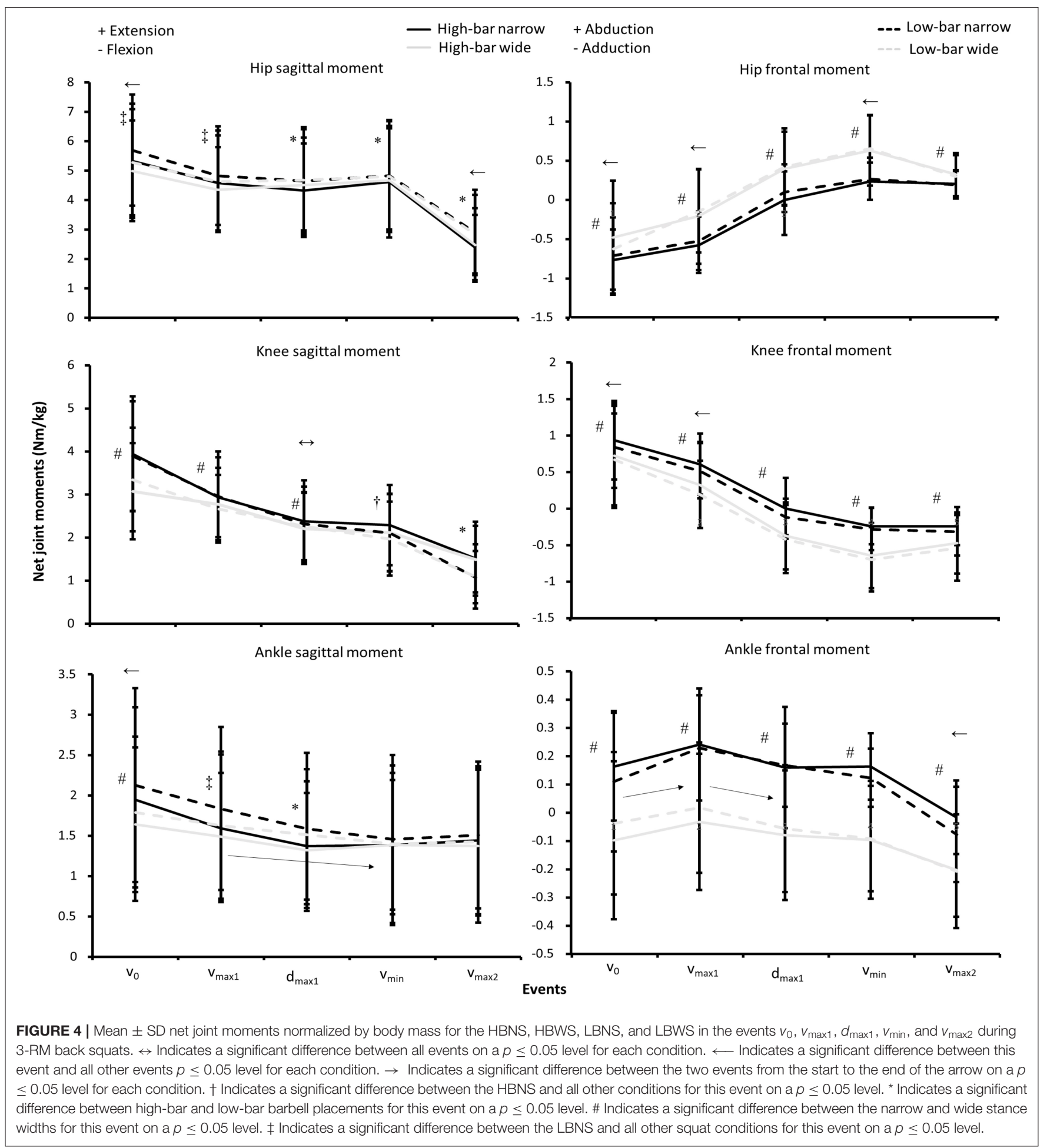

contributions during all events compared with the wide stance widths. Knee contribution decreased from $v_{0}$ to all other events for all squat conditions $(p \leq 0.024)$. The HBNS had a greater knee contribution than all other squat conditions in $v_{0}$ while similar knee contributions were produced at $v_{\max 1}$ and $d_{\max 1}$. At $v_{\min }$ and $v_{\max 2}$, both high-bar placements had a greater knee contribution than the low-bar placements. Ankle contributions were stable throughout the four first events before increasing at $v_{\max 2}(p \leq 0.001)$, where the LBNS had a greater ankle contribution at $v_{0}$ and $v_{\max 1}$ than all other squat conditions. The narrow stance width had a greater ankle contribution at $d_{\max }$ and $v_{\min }$ than the wide stance widths before decreasing at $v_{\max 2}$. 


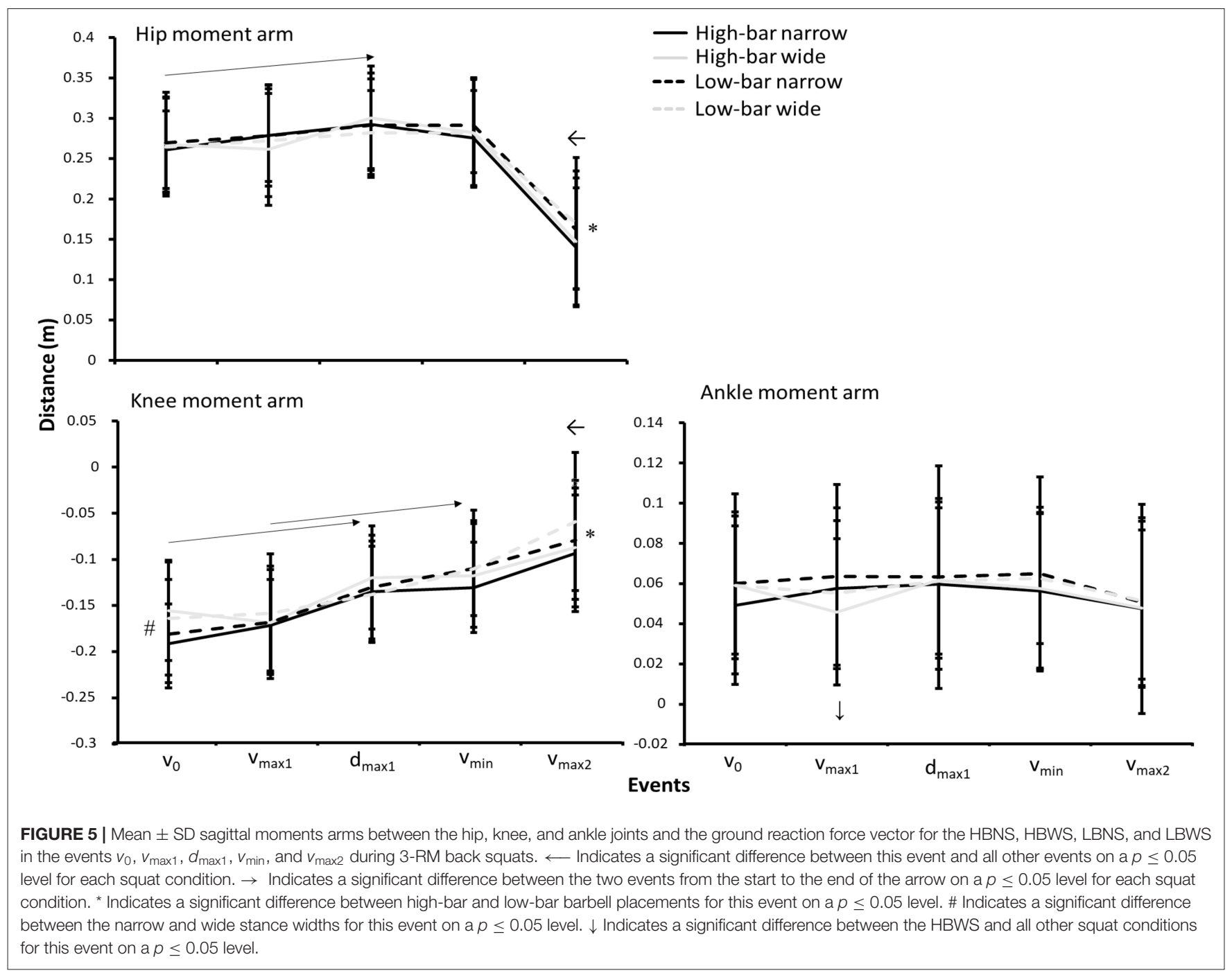

The myoelectric activity was significantly different in the sticking region for all muscles $\left(F \geq 12.93, p \leq 0.002, \eta^{2} \geq 0.46\right)$ except for the adductor longus and gluteus medius $(F \leq 1.58, p \geq$ $\left.0.218, \eta^{2} \leq 0.067\right)$. A significant effect of stance width was found for vastus lateralis activity $\left(F=4.9, p=0.038, \eta^{2}=0.20\right)$. Also, a significant interaction between barbell placement and stance width was observed for vastus lateralis and gluteus maximus ( $F$ $\geq 4.62, p \leq 0.044, \eta^{2} \geq 0.19$ ). Finally, a significant interaction between the sticking region and the stance width occurred for soleus and gastrocnemius activity $\left(F \geq 4.62, p \leq 0.041, \eta^{2} \geq 0.24\right.$; see Figures 7, 8).

Post-hoc tests showed that myoelectric activity for the erector spinae decreased from the sticking region to the poststicking region for all squat conditions $(p=0.011)$, whereas the myoelectric activity of quadriceps decreased from the presticking and sticking region to the poststicking region for all squat conditions $(p \leq 0.025)$. Furthermore, the narrow stance widths resulted in greater vastus lateralis myoelectric activity than the wide stance widths during the presticking region. A barbell placement stance width interaction effect indicated that the
HBNS demonstrated greater vastus lateralis myoelectric activity during the poststicking region compared with all other squat conditions $(p=0.044)$. Squatting with a narrow stance width produced greater gastrocnemius and soleus myoelectric activity in the presticking region. In the sticking and poststicking regions, gastrocnemius and soleus myoelectric activity decreased, as indicated by the sticking point region stance width significant interaction effect. However, the opposite pattern was observed for the gluteus maximus and hamstring muscles, as their activity increased significantly during each sticking point region $(p \leq$ 0.038). Finally, the HBNS squat produced less gluteus maximus myoelectric activity in the presticking region compared with all the other squat conditions, indicated by the significant barbell placement stance width interaction effect.

\section{DISCUSSION}

The main findings from this study were that the sticking region started at a lower barbell height for the HBWS compared with 


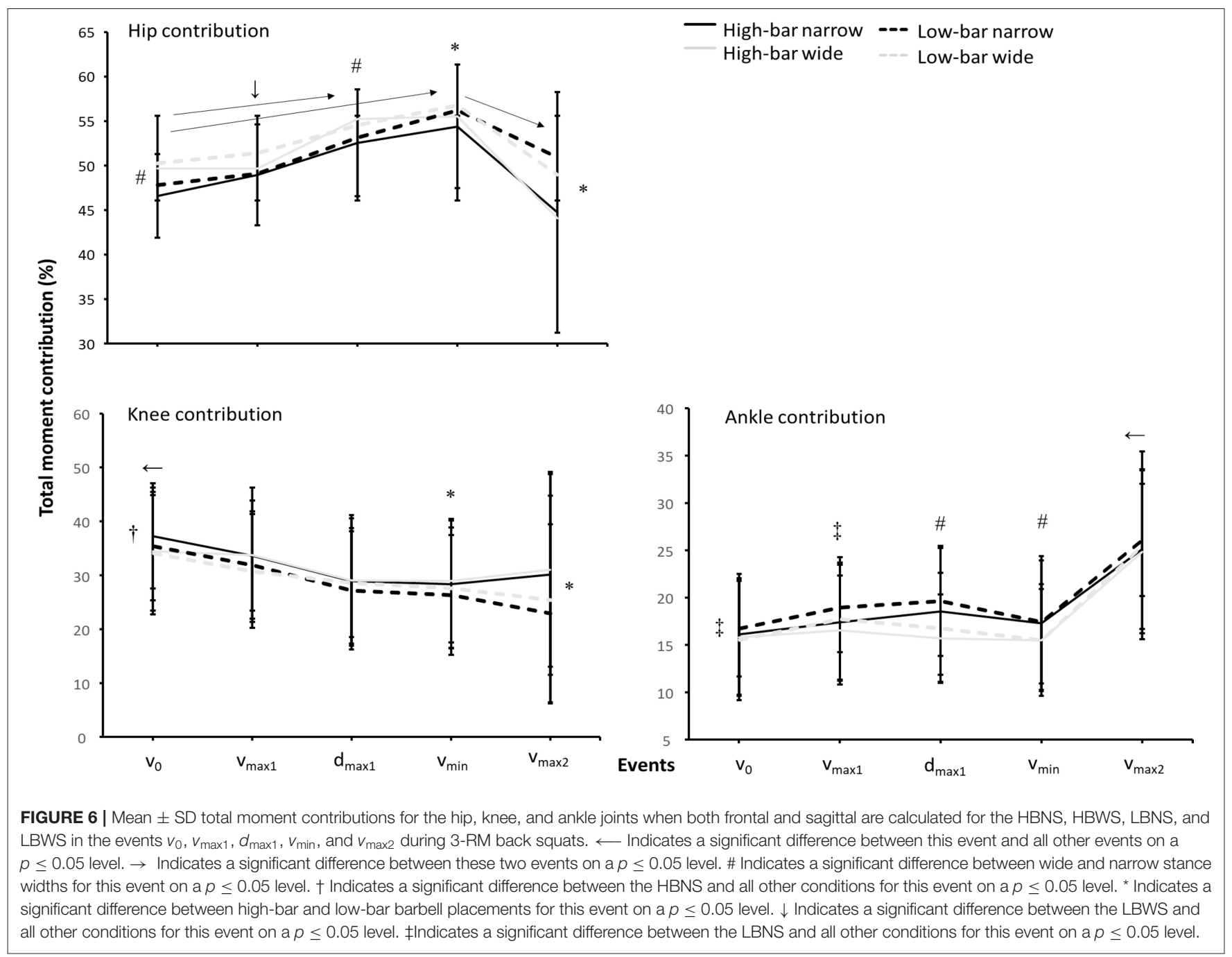

the other squat conditions. Squatting with an HBNS resulted in deeper knee flexion angles than the other squat conditions, and therefore greater squat depth, whereas the low-bar conditions resulted in larger torso inclination at all events. All squat conditions resulted in medially directed ground reaction forces, but to a greater extent with the wide stance width, which increased for all squat conditions in $v_{\min }$. Furthermore, the knee moment arm decreased during all events, whereas the hip moment arm peaked in $d_{\max 1}$ and $v_{\min }$, independent of squat condition. Also, the hip joint was responsible for over $50 \%$ of the total moment contributions at $d_{\max 1}$ and $v_{\min }$ for all squat conditions. This finding confirms our hypothesis that the large hip demands around the sticking region may be the main limitation for overcoming it, independent of squat condition. Squatting with an LBWS resulted in greater hip contributions than the other squat conditions, partly confirming our second hypothesis that the low-bar conditions would produce greater hip contributions than the high-bar conditions. Finally, between the squat conditions, vastus lateralis and gastrocnemius myoelectric activity were greater for the narrow stance widths than the wide stance width, whereas gluteus maximus activity was lower for the HBNS than the other squat conditions.

Our findings showed that peak moments were produced at $v_{0}$, whereas in the sticking region it started at around 0.15$0.17 \mathrm{~m}$ barbell height and at $0.25-0.27 \mathrm{~s}$. This may be explained by muscle potentiation of the quadriceps, caused by the stretchshortening cycle, which makes it possible to produce more force during the presticking region, as observed in the study of van den Tillaar et al. (2021). The potentiation effect, has in previous studies, been reported to dimmish after around $0.3 \mathrm{~s}$, which is around where the sticking region started for all squat conditions.

Furthermore, the concentric ascent starts with both knee extension and plantar flexion, and there was decreased myoelectric activity from the presticking and sticking to the poststicking region in the quadriceps and plantar flexors for all squat conditions. Therefore, strengthening these muscles should not be neglected, as their greater contribution could result in $d_{\max 1}$ occurring at a higher vertical barbell height, increasing the chance of a successful lift. Moreover, because ground reaction force was at its lowest in $d_{\max 1}$ due to peak deacceleration, it 


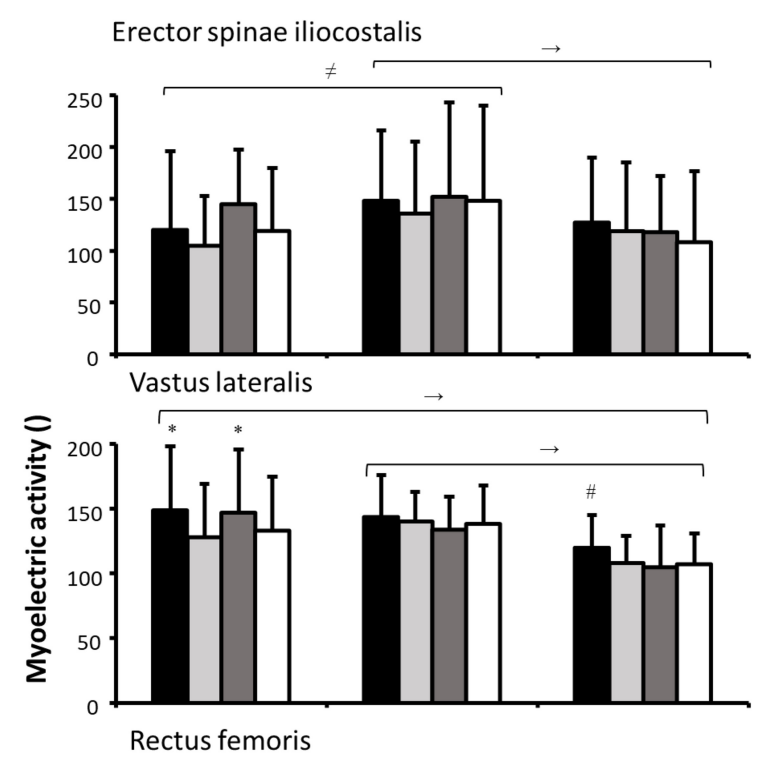

Erector spinae longissimus

- High-bar narrow

- High-bar wide

- Low-bar narrow

- Low-bar wide
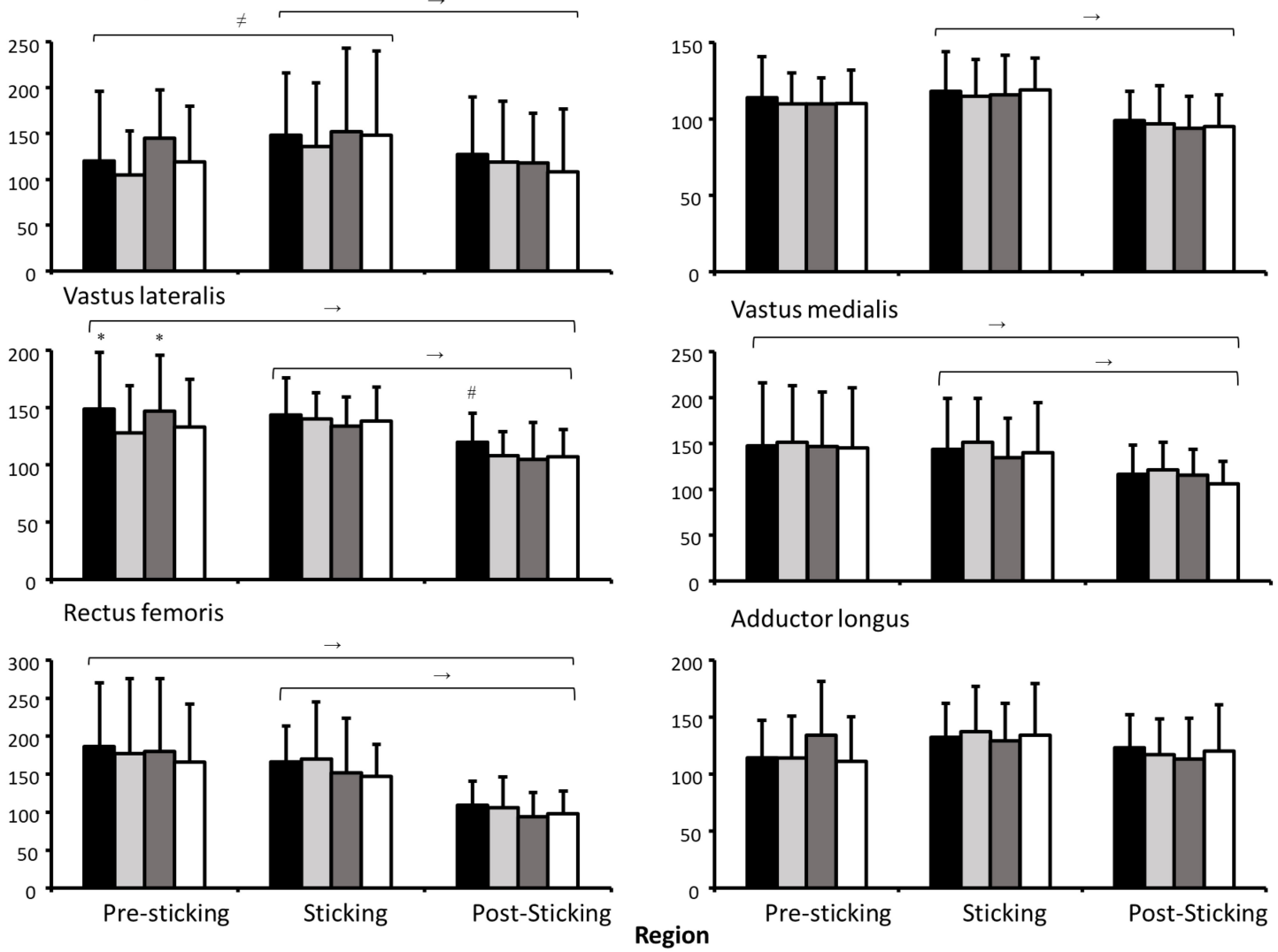

FIGURE 7 | Mean \pm SD normalized myoelectric activity for the erector spinae iliocostalis and longissimus, vastus lateralis and medialis, rectus femoris, and adductor longus during 3-RM HBNS, HBWS, LBNS, and LBWS in the presticking, sticking, and poststicking regions. $\rightarrow$ Indicates a significant difference between these two regions for all conditions on a $p \leq 0.05$ level. $\neq$ Indicates a significant difference for the HBNS, HBWS, and LBWS between these two regions on a $p \leq 0.05$ level. * Indicates a significant difference between this condition and all other stance widths for this region on a $p \leq 0.05$ level. \# Indicates a significant difference between this condition and all other squat conditions in this region on a $p \leq 0.05$ level.

demonstrates that this is the event in the lift where the capability of the lifter to produce force is at its lowest, independent of squat condition. Therefore, we suggest that authors avoid characterizing $v_{\text {min }}$ (when velocity is lowest) as the sticking point and instead, refer to $d_{\max }$ (where deacceleration is highest) as the sticking point, independent of the squat type or stance.

The participants squatted the greatest load with the LBNS followed by the HBNS, the LBWS, and then the HBWS. When squatting with an HBWS, the sticking region started and ended at around $0.03-0.04 \mathrm{~m}$ lower than the barbell height of the other squat conditions (Figure 1). Moreover, the HBWS increased forward lean by $2.4^{\circ}$ from $v_{0}$ to $v_{\max 1}$, whereas the other conditions increased forward lean by $4.3^{\circ}-4.4^{\circ}$ at the same events. This reflected a different development from $v_{0}$ to $v_{\max 1}$ for the hip, knee, and ankle moment arms (Figure 5), where the hip and ankle moment arm decreased and the knee moment arm increased for the HBWS whereas all other squat conditions showed the opposite pattern. It is speculated that the combination of the lower barbell height for the HBWS from $v_{0}$ compared with the other squat conditions, together with a smaller forward lean at the start of the sticking region, reduces the contribution of the hip extensors to the hip extensor moment. This may be because an increased forward lean lengthens the hip extensors, increasing the ability of these muscles to generate the force (Escamilla et al., 2001).

Furthermore, squatting with an LBNS resulted in the participants lifting $>3 \mathrm{~kg}$ greater loads than the other squat conditions. This was in contrast with the findings reported by Lahti et al. (2019), who reported no significant differences in the load lifted between stance widths. This may be explained by two factors. First, squatting with an LBNS resulted in a greater hip extension moment at $v_{0}$ and $v_{\max 1}$ than the other squat conditions. It may be that putting greater demands on the hip extensors at the beginning of the ascent could be advantageous 


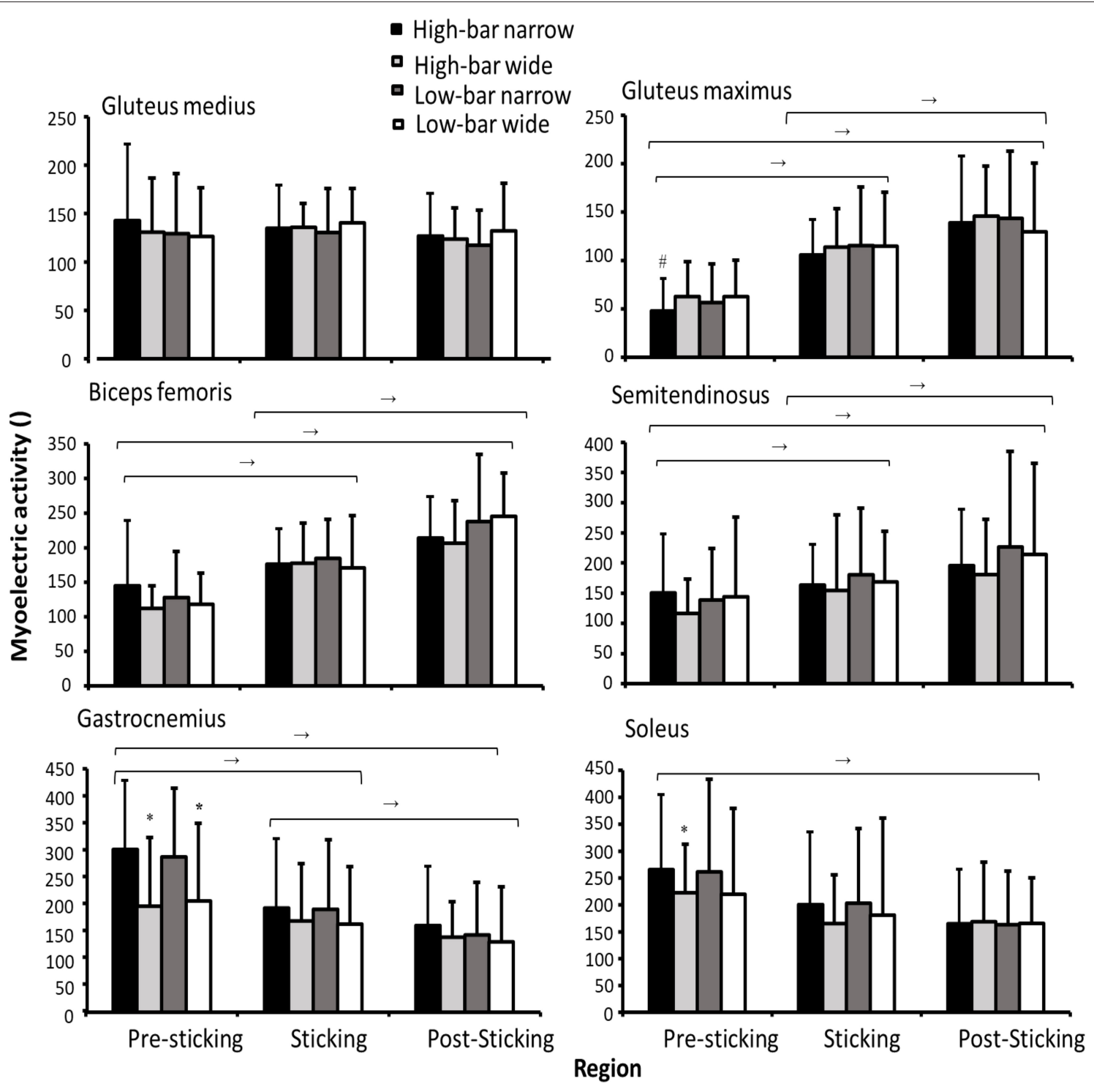

FIGURE 8 | Mean \pm SD normalized myoelectric activity for the gluteus medius and maximus, biceps femoris, semitendinosus, gastrocnemius, and soleus during 3-RM HBNS, HBWS, LBNS, and LBWS in the presticking, sticking, and poststicking regions. $\rightarrow$ Indicates a significant difference between these two regions for all conditions on a $p \leq 0.05$ level. * Indicates a significant difference between this condition with other stance widths for this region on a $p \leq 0.05$ level. * Indicates a significant difference between the HBNS and all other conditions for this region on a $p \leq 0.05$ level.

when the goal is to lift the greatest loads possible because the myoelectric activity of the quadriceps has been reported to not change much between $>50$ and $90 \%$ of 1-RM (van den Tillaar et al., 2019), implying that the quadriceps may already be nearmaximal activation at lower percentages of 1-RM. Therefore, choosing a technique that places more demand on hip extension moments could enable greater loads to be lifted. Second, participants in the study of Lahti et al. (2019) squatted with $\sim 1.0$ and 1.5 times greater trochanter width for the narrow and wide stance widths. The participants in the current study squatted with 0.7 and 1.7 shoulder width, resulting in the mediolateral to vertical force ratio being $\sim 50 \%$ higher for the wide stance widths compared with the narrow stance widths, and thereby, producing 1.5 to 2 times higher laterally-directed forces for the wide stance widths than the narrow stance widths (Figure 3). This created a superior medially-directed resultant force vector and thereby, greater hip abduction together with knee and ankle adduction moments for the wide stance widths. More importantly, our data indicate that squatting with 1.7 times shoulder width results in around $50 \%$ greater laterally-directed forces exerted against the ground for the wide stance width. Moreover, the larger hip abduction moment for the wide stance widths resulted in greater hip contributions to the total moment for the wide stance widths, and especially the LBWS. The larger hip contributions were 
a result of lower knee extension and plantar flexion moments during the presticking region together with greater hip abduction moments in the sticking region for the wide stance widths (Figure 4). It is speculated that the larger hip abduction and knee adduction moments together with the greater mediolateral to vertical force ratios around the sticking region for the wide stance widths resulted in a less effective vertical lifting technique, despite greater hip contributions compared with the narrow stance widths. Therefore, our findings suggest that the LBWS, typically referred to as a hip dominant squat condition in the literature (Glassbrook et al., 2017), enables greater hip contributions to the total extensor moment in the sticking region because of the hip abduction moments.

The vastus lateralis produced greater myoelectric activity during the narrow stance widths and during the post-sticking region for the HBNS compared with all other squat conditions. Since the HBNS demonstrated $5^{\circ}-7^{\circ}$ greater peak knee flexion than the wide stance widths together with $3^{\circ}-4^{\circ}$ greater flexion at the start of the post-sticking region, the increased knee flexion angle during these regions probably caused increased mechanical work and thereby increased vastus lateralis myoelectric activity. This is supported by Bryanton et al. (2012) who investigated both squat depth and barbell load on the relative hip, knee, and ankle muscular effort between 50 and $90 \%$ of 1 -RM in back squats and found that the knee extensor relative muscle effort (the ratio of net joint moment to maximum voluntary torque, matched for joint angle) increased with deeper knee flexion angles, but not barbell load. The HBNS produced less gluteus maximus activity in the presticking region compared with other squat conditions, which is logical since increased depth lengthens the gluteus and reduces its capability to produce force (Vigotsky and Bryanton, 2016). However, no significant differences were observed in myoelectric activity for the vastus medialis. It is speculated that the vastus medialis was maximally activated for all squat conditions, and during the wide stances, the inability to produce additional myoelectric activity resulted in greater hip internal rotation and, thereby, a greater knee valgus for the wide stance widths. For the shank, plantar flexion moments were greater while performing back squats with a narrow stance width during the presticking region (Figure 4). This occurred since ankle flexion angles were $4^{\circ}-9^{\circ}$ greater during all events for the narrow stance widths and were not influenced much by barbell placement. Similar findings were reported by Swinton et al. (2012), who found that peak ankle flexion was $\sim 10^{\circ}$ greater for narrow stance widths, whereas the present study reported $5^{\circ}-7^{\circ}$ greater ankle flexion for the narrow stance.

Furthermore, larger spinal erector myoelectric activity was observed in the sticking region compared with the poststicking region for all squat conditions. Also, greater myoelectric activity was observed in the presticking region compared with the poststicking region for the erector spinae iliocostalis muscle. These muscles are especially important in the squats because they help to maintain anteroposterior spinal integrity, providing a contribution to spinal stabilization (Schoenfeld, 2010). The higher erector spinae myoelectric activity during the sticking region was probably observed because of the increased forward lean at this point of the lift, resulting in greater hip moment arms and increasing the demands for the erector spinae to contribute to spinal stabilization. This finding is in accordance with a previous study that observed increased myoelectric activity when forward lean increased, compared with a neutral position (Zimmermann et al., 1993).

\section{LIMITATIONS AND FURTHER DIRECTIONS}

The participants in this study were recreationally trained lifters and not powerlifters or strength athletes. Therefore, our findings may not be generalizable to powerlifters or strength athletes. Also, the present study only reported the net joint forces calculated and resultant moments from inverse dynamics analyses, and not the joint contact forces (Vigotsky et al., 2019). This method neglects muscle forces, which often are the primary sources of joint loading (Vigotsky et al., 2019). Therefore, further research should use musculoskeletal modeling techniques to quantify the joint contact force.

\section{CONCLUSION AND PRACTICAL APPLICATION}

Squatting with an LBWS produced greater hip contributions to the total moment, whereas squatting with an HBNS resulted in deeper knee flexion angles and higher knee contributions to the total moment, together with less gluteus maximus and higher vastus lateralis myoelectric activity. Therefore, our findings suggest that training with an HBNS could be beneficial when targeting the knee extensors and plantar flexors, whereas an LBWS could be beneficial when targeting the hip extensors.

\section{DATA AVAILABILITY STATEMENT}

The raw data supporting the conclusions of this article will be made available by the authors, without undue reservation.

\section{ETHICS STATEMENT}

The studies involving human participants were reviewed and approved by Norwegian Center for Research Data project number 701688. The patients/participants provided their written informed consent to participate in this study.

\section{AUTHOR CONTRIBUTIONS}

All authors were involved in planning the study, while EK and SL performed the data collection and analysis. SL wrote the first draft, while the other three authors others discussed and rewrote the manuscript. RT was the supervisor of the project. All authors contributed to the article and approved the submitted version. 


\section{REFERENCES}

Anderson, R., Courtney, C., and Carmeli, E. (1998). EMG analysis of the vastus medialis/vastus lateralis muscles utilizing the unloaded narrow-and widestance squats. J. Sport Rehabil. 7, 236-247. doi: 10.1123/jsr.7.4.236

Bell, A. L., Brand, R. A., and Pedersen, D. R. (1987). Prediction of hip joint center location from external landmarks. J. Biomech. 20:913. doi: 10.1016/0021-9290(87)90226-0

Bell, A. L., Pedersen, D. R., and Brand, R. A. (1990). A comparison of the accuracy of several hip center location prediction methods. J. Biomech. 23, 617-621. doi: 10.1016/0021-9290(90)90054-7

Benz, R., and West Chester, P. (1989). A Kinematic Analysis of the High and Low Bar Squat Techniques by Experienced Low Bar Weight Lifters. West Chester, PA: West Chester University.

Bishop, P., Cureton, K., and Collins, M. (1987). Sex difference in muscular strength in equally-trained men and women. Ergonomics 30, 675-687. doi: $10.1080 / 00140138708969760$

Bryanton, M. A., Kennedy, M. D., Carey, J. P., and Chiu, L. Z. (2012). Effect of squat depth and barbell load on relative muscular effort in squatting. J. Strength Cond. Res. 26, 2820-2828. doi: 10.1519/JSC.0b013e31826791a7

C-Motion (2017). Marker Set Guidelines. C-Motion Wiki Documentation.

Cohen, J. (1988). Statistical Power Analysis for the Behavioral Sciences. Hillsdale, NJ: Erbaum Press.

Elliott, B., Wilson, G., and Kerr, G. (1989). A biomechanical analysis of the sticking region in the bench press. Med. Sci. Sports Exerc. 21, 450-462. doi: 10.1249/00005768-198908000-00018

Escamilla, R. F., Fleisig, G. S., Lowry, T. M., Barrentine, S. W., and Andrews, J. (2001). A three-dimensional biomechanical analysis of the squat during varying stance widths. Med. Sci. Sports Exerc. 33, 984-998. doi: 10.1097/00005768-200106000-00019

Fry, A., Aro, T., Bauer, J., and Kraemer, W. (1993). A comparison of methods for determining kinematic properties of three barbell squat exercises. J. Hum. Mov. Stud. 24:83.

Glassbrook, D. J., Brown, S. R., Helms, E. R., Duncan, S., and Storey, A. G. (2019). The high-bar and low-bar back-squats: a biomechanical analysis. J. Strength Cond. Res. 33, S1-S18. doi: 10.1519/JSC.0000000000001836

Glassbrook, D. J., Helms, E. R., Brown, S. R., and Storey, A. G. J. (2017). A review of the biomechanical differences between the high-bar and low-bar back-squat. J. Strength Cond. Res. 31, 2618-2634. doi: 10.1519/JSC.0000000000002007

Greig, L., Hemingway, B. H. S., Aspe, R. R., Cooper, K., Comfort, P., and Swinton, P. A. (2020). Autoregulation in resistance training: addressing the inconsistencies. Sports Med. 2020, 1-15. doi: 10.1007/s40279-020-01330-8

Hermens, H. J., Freriks, B., Disselhorst-Klug, C., and Rau, G. (2000). Development of recommendations for SEMG sensors and sensor placement procedures. J. Electromyogr. Kinesiol. 10, 361-374. doi: 10.1016/S1050-6411(00)00 $027-4$

IP Federation (2019). International Powerlifting Federation.Technical Rules Book.

Lahti, J., Hegyi, A., Vigotsky, A. D., and Ahtiainen, J. P. (2019). Effects of barbell back squat stance width on sagittal and frontal hip and knee kinetics. Scand. J. Med. Sci. Sports 29, 44-54. doi: 10.1111/sms.13305

Larsen, S., Kristiansen, E., and van den Tillaar, R. (2021a). Effects of subjective and objective autoregulation methods for intensity and volume on enhancing maximal strength during resistance-training interventions: a systematic review. PeerJ.

Larsen, S., Kristiansen, E., and van den Tillaar, R. (2021b). New insights about the sticking region in back squats: an analysis of kinematics, kinetics, and myoelectric activity. Front. Sports Act. Liv. 3:691459. doi: 10.3389/fspor.2021.691459

Maddox, E. U., Sievert, Z. A., and Bennett, H. J. (2020). Modified vector coding analysis of trunk and lower extremity kinematics during maximum and sub-maximum back squats. J. Biomech. 106:109830. doi: 10.1016/j.jbiomech.2020.109830

McCaw, S., and Melose, D. (1999). Stance width and bar load effects on leg muscle activity during the parallel squat. Med. Sci. Sports Exerc. 31, 428-436.

Schoenfeld, B. J. (2010). Squatting kinematics and kinetics and their application to exercise performance. J. Strength Cond. Res. 24, 3497-3506. doi: 10.1519/JSC.0b013e3181bac2d7

Swinton, P. A., Lloyd, R., Keogh, J. W., Agouris, I., and Stewart, A. D. (2012). A biomechanical comparison of the traditional squat, powerlifting squat, and box squat. J. Strength Cond. Res. 26, 1805-1816. doi: 10.1519/JSC.0b013e3182577067

van den Tillaar, R., Andersen, V., and Saeterbakken, A. H. (2019). Comparison of muscle activation and kinematics during free-weight back squats with different loads. PLoS ONE 14:e0217044. doi: 10.1371/journal.pone.0217044

van den Tillaar, R., Knutli, T., and Larsen, S. (2020). The effects of barbell placement on kinematics and muscle activation around the sticking region in squats. Front. Sports Act. Liv. 2:172. doi: 10.3389/fspor.2020.604177

van den Tillaar, R., Kristiansen, E. L., and Larsen, S. (2021). Is the occurrence of the sticking region in maximum smith machine squats the result of diminishing potentiation and co-contraction of the prime movers among recreationally resistance trained males? Int. J. Environ. Res. Public Health 18:1366. https://doi. org/10.3390/ijerph18031366

van den Tillaar, R., and Larsen, S. (2020). Kinematic and EMG comparison between variations of unilateral squats under different stabilities. Sports Med. Int. Open 4:E59. doi: 10.1055/a-1195-1039

Vigotsky, A., and Bryanton, M. (2016). "Relative muscle contributions to net joint moments in the Barbell back squat", in: American Society of Biomechanics 40th Annual Meeting. Raleigh, NC.

Vigotsky, Zelik, K. E., Lake, J., and Hinrichs, R.N. (2019). Mechanical Misconceptions: Have we lost the "mechanics" in "sports biomechanics"? J. Biomech. 93, 1-5. doi: 10.1016/j.jbiomech.2019.07.005

Wretenberg, P., Feng, Y., and Arborelius, U. (1996). High-and low-bar squatting techniques during weight-training. Med. Sci. Sports Exerc. 28, 218-224. doi: 10.1097/00005768-199602000-00010

Zimmermann, C. L., Cook, T. M., and Goel, V. K. (1993). Effects of seated posture on erector spinae EMG activity during whole body vibration. Ergonomics 36, 667-675. doi: 10.1080/00140139308967928

Zourdos, M. C., Klemp, A., Dolan, C., Quiles, J. M., Schau, K. A., Jo, E., et al. (2016). Novel resistance training-specific rating of perceived exertion scale measuring repetitions in reserve. J. Strength Cond. Res. 30, 267-275. doi: $10.1519 /$ JSC. 0000000000001049

Conflict of Interest: The authors declare that the research was conducted in the absence of any commercial or financial relationships that could be construed as a potential conflict of interest.

Publisher's Note: All claims expressed in this article are solely those of the authors and do not necessarily represent those of their affiliated organizations, or those of the publisher, the editors and the reviewers. Any product that may be evaluated in this article, or claim that may be made by its manufacturer, is not guaranteed or endorsed by the publisher.

Copyright $\odot 2021$ Larsen, Kristiansen, Helms and van den Tillaar. This is an openaccess article distributed under the terms of the Creative Commons Attribution License (CC BY). The use, distribution or reproduction in other forums is permitted, provided the original author(s) and the copyright owner(s) are credited and that the original publication in this journal is cited, in accordance with accepted academic practice. No use, distribution or reproduction is permitted which does not comply with these terms. 